\title{
Validation of a Carotid Intima-Media Thickness Border Detection Program for Use in an Office Setting
}

\author{
Adam D. Gepner, BS, Claudia E. Korcarz, DVM, RDCS, \\ Susan E. Aeschlimann, RDMS, RVT, Tamara J. LeCaire, MS, Mari Palta, PhD, \\ Wendy S. Tzou, MD, and James H. Stein, MD, FASE, Madison, Wisconsin
}

\begin{abstract}
Background: A unique semiautomated border detection program (BDP) designed for use on a personal computer was evaluated to determine whether: (1) carotid intima-media thickness (CIMT) measurements were bioequivalent to a reference laboratory; and (2) it would allow a novice (NOV) reader with no medical training to accurately and reproducibly measure CIMT.

Methods: Far-wall CIMT was measured blindly and in duplicate by an experienced and NOV reader using BDP and by a reference laboratory.
\end{abstract}

$\mathrm{U}$ thickness (CIMT) is a noninvasive and reproducible method to detect and quantify subclinical atherosclerosis. Autopsy studies have demonstrated a direct histologic relationship between carotid and coronary atherosclerosis. ${ }^{1,2}$ CIMT measurements have been used to measure differences in the progression of atherosclerosis in clinical trials. ${ }^{3}$ In observational trials, increased CIMT is associated with prevalent and incident cardiovascular disease. ${ }^{4-6} \mathrm{Al}-$ though ultrasound measurement of CIMT has been recommended as a clinical screening tool, its use has been limited to research studies or selected institutions, in part because of labor- and time-intensive measurement protocols. ${ }^{7}$ An analysis package that allows straightforward and efficient measurement of

From the Division of Cardiovascular Medicine, Department of Medicine, and the Department of Population Health Sciences, University of Wisconsin Medical School.

Supported in part by the National Center for Research Resources (K23 RR16176-01), and the National Heart, Lung, and Blood Institute (R01 HL62897). Dr Tzou was supported by a National Institutes of Health National Service Award (T32 HL07936) from the University of Wisconsin Cardiovascular Research Center. Sonosite Inc provided the software used in this study.

Disclosure: Dr Stein has received research grants from Siemens Medical Solutions, Camtronics Medical Systems, and Sonosite.

Reprint requests: James H. Stein, MD, Section of Cardiovascular Medicine, Department of Medicine, University of Wisconsin Medical School, 600 Highland Ave, G4/341 CSC (MC 3248), Madison, WI 53792 (E-mail: jbs@medicine.wisc.edu).

$0894-7317 / \$ 32.00$

Copyright 2006 by the American Society of Echocardiography. doi:10.1016/j.echo.2005.09.006
Results: Mean CIMT using BDP was bioequivalent to the reference laboratory (two 1 -sided T-test, $P<.05$ ) with small absolute differences (experienced $0.011 \pm 0.004$ $\mathrm{mm}$, NOV $0.022 \pm 0.004 \mathrm{~mm})$. Reproducibility was high, with small coefficients of variation when used by either the experienced $(3.1 \%)$ or NOV $(7.8 \%)$ reader.

Conclusion: CIMT measurements using BDP were accurate and reproducible. It was mastered easily by a NOV reader and appeared suitable for use in an office setting. (J Am Soc Echocardiogr 2006;19:223-228.)

CIMT, while maintaining accuracy and reproducibility, could be a useful clinical tool.

This study evaluated a new, semiautomated border detection program (BDP), designed for use on a personal computer or handheld ultrasound system. Measurements were compared with a reference (REF) laboratory to determine bioequivalence and performed in duplicate by an experienced (EXP) and novice (NOV) reader to evaluate intraobserver reproducibility and to assess whether BDP would allow a NOV reader with no medical or ultrasound training to accurately and reproducibly measure CIMT.

\section{METHODS}

\section{Experimental Protocol}

Our institutional review board approved this study. Data were obtained from 25 consecutive healthy individuals (mean [SD] age 28.0 [7.7] years) who were imaged as part of the Cardiovascular Disease in Type 1 Diabetes Study. Images from an additional 15 consecutive participants (age 55.2 [7.6] years) provided a wider range of CIMT values for this validation study. These patients had been referred by their physicians to our Vascular Health Screening Program for Determination of CIMT.

\section{Carotid Ultrasound Images}

B-mode ultrasound images of the distal $1 \mathrm{~cm}$ of the common carotid artery (CCA) were obtained using an 8.0-MHz linear-array transducer (8L5, Acuson Sequoia, 


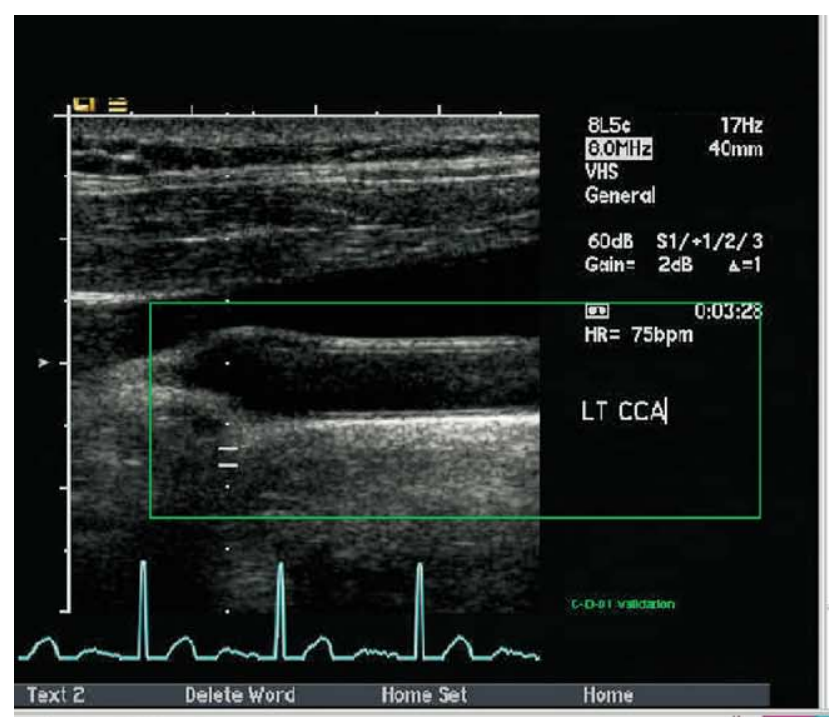

MANUAL SLOPE: Choose Point 1

Calibrated. Pixe1 Size $=0.116279 \mathrm{~mm}$

Mean Thickness: $0.629 \mathrm{~mm}$

Thickest $1.0 \mathrm{~mm}$ Region: $0.697 \mathrm{~mm}$

Standard deviation $=0.0625 \mathrm{~mm}$

Standard error of the mean $=0.00678 \mathrm{~mm}$

Measure Extent $=9.884 \mathrm{~mm}$ ( 85 pizels)

Patient CD-1 ag2

Gender, Ethnicity: Male, White/Other Age: 104

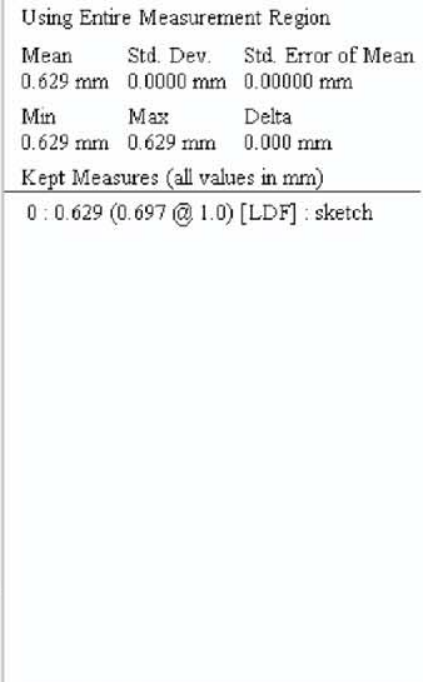

sing Entire Measurement Region

Std Dev. Std Error of Mean

Min May Delta

$0.629 \mathrm{~mm} \quad 0.629 \mathrm{~mm} \quad 0.000 \mathrm{~mm}$

Kept Measures (all values in mm)

$0: 0.629(0.697 @ 1.0)[\mathrm{LDF}]:$ sketch
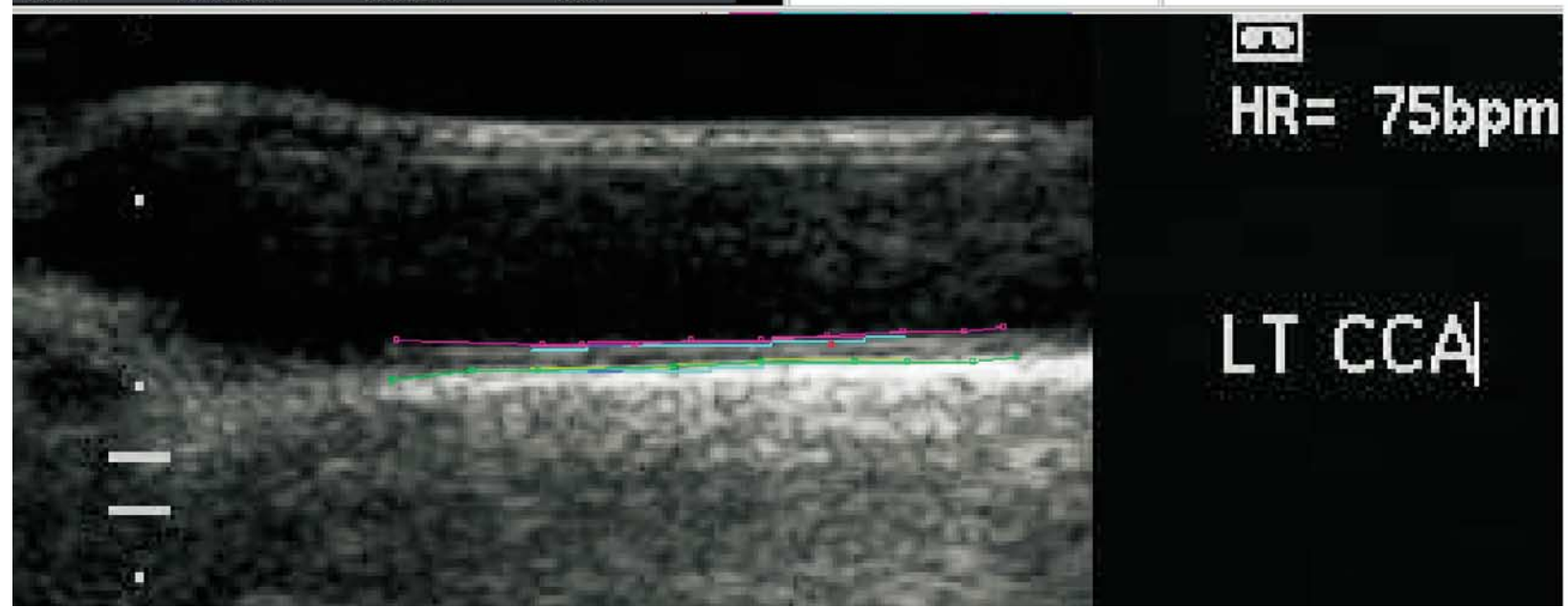

Figure 1 Semiautomated measurement of far-wall common carotid artery intima-media thickness using border detection program.

Siemens Medical Solutions, Mountain View, Calif). Image acquisition was based on a standardized protocol from the Atherosclerosis Risk in Communities Study, but was limited to only the CCA. ${ }^{8}$ Images were obtained from 3 different angles: an optimal angle of incidence defined as the longitudinal angle of approach where both branches of the internal and external carotid arteries are visualized simultaneously; an anterior angle; and a posterior angle. All images were obtained by a single sonographer (S. E. A.). Images were recorded and stored digitally from the analogue video output and converted to bitmaps using software (Vericis for Echocardiography, Camtronics Medical Systems, Hartland, Wis). Videotape was not used. Resolution was limited by the ultrasound system and transducer, not the format or manipulation of the images, and was constant at $\mathbf{8 5 . 6 7}$ pixels/cm $(0.11 \mathrm{~mm} /$ pixel $)$.

\section{CIMT Measurements}

Mean and maximum far-wall CIMT of each CCA were measured blindly and in duplicate by an EXP and a NOV reader using BDP (SonoCalc IMT, Sonosite Inc, Bothell, Wash). The EXP reader (C. E. K.), a RDCS with 19 years of ultrasound experience, was the manager of our atherosclerosis imaging research program laboratory, and had 3 years of experience performing and reading CIMT studies. The NOV reader (A. D. G.) was a premedical student with no previous ultrasound experience except for a half-day training session and 5 practice readings before starting this study. Each set of images was measured on two separate occasions using a semiautomated BDP (SonoCalc IMT) (Figure 1). Each reader was blinded to their previous measurements and to those of the other reader. The semiautomated BDP traced a specific region of interest based on image intensity, vessel morphology, and pre- 
Table 1 Comparisons with reference laboratory for mean and maximum common CIMT thickness measurements

\begin{tabular}{lcc}
\hline & Experienced reader & Novice reader \\
\hline $\begin{array}{l}\text { Mean CIMT } \\
\text { Mean absolute }\end{array}$ & & \\
$\quad$ difference, $\mathrm{mm}$ & $0.011 \pm 0.004$ & $0.022 \pm 0.004$ \\
$P$ & $.954(<.001)$ & $.955(<.001)$ \\
Maximum CIMT & & \\
$\quad$ Mean absolute & & \\
$\quad$ difference, $\mathrm{mm}$ & $-0.031 \pm 0.007$ & $-0.011 \pm 0.006$ \\
$P$ & $.914(<.001)$ & $.921(<.001)$ \\
\hline
\end{tabular}

CIMT, Carotid intima-media thickness.

defined segment length $(1 \mathrm{~cm})$ after the user determined the segment of interest by a single mouse click. The blood-intima and media-adventitia borders within the region of interest were automatically detected and smoothed borders were created. If the reader decided that the automated border was not acceptable, the user could modify with a series of mouse clicks along each border. A new vessel outline was generated based on these edits, along with calculated mean and maximum CIMT values. Each CCA segment was measured in triplicate and segmental values were calculated as the average of those 3 measurements. The time to make measurements for each patient was recorded by each reader. Finally, additional, blinded readings were performed by an independent REF laboratory (AUTREC, Raleigh, NC). ${ }^{9}$

\section{Statistical Techniques}

All values were reported as mean \pm SEM. Intraobserver reproducibility was analyzed using paired $t$ tests and linear regression. Coefficients of variation $(\mathrm{CV})$ were determined by taking the SD divided by the mean using the root mean square approach. ${ }^{10}$ Comparisons of the first of NOV and EXP readings using BDP with the REF laboratory were analyzed using Bland-Altman plots and linear regression. ${ }^{11}$ Mean differences and bioequivalence were tested using the 2 1-sided $t$ test (TOST) approach. ${ }^{12,13}$ Predetermined levels of acceptable differences between readers and the REF laboratory were 1 digital pixel $(0.11 \mathrm{~mm})$ for mean CIMT and 2 digital pixels $(0.22 \mathrm{~mm})$ for maximum CIMT.

\section{RESULTS}

\section{Participant Characteristics}

Images from 40 participants ( 14 men, 26 women) were analyzed. Their mean age was $38.2 \pm 2.4$ years (range 18-68 years). Based on the REF laboratory measurements, the average mean CIMT of the participants was $0.613 \pm 0.013 \mathrm{~mm}$ and the average maximum CIMT was $0.694 \pm 0.014 \mathrm{~mm}$.

\section{Comparison with REF Laboratory}

Using the BDP, measurements from both the EXP and NOV readers were comparable and bioequiva-

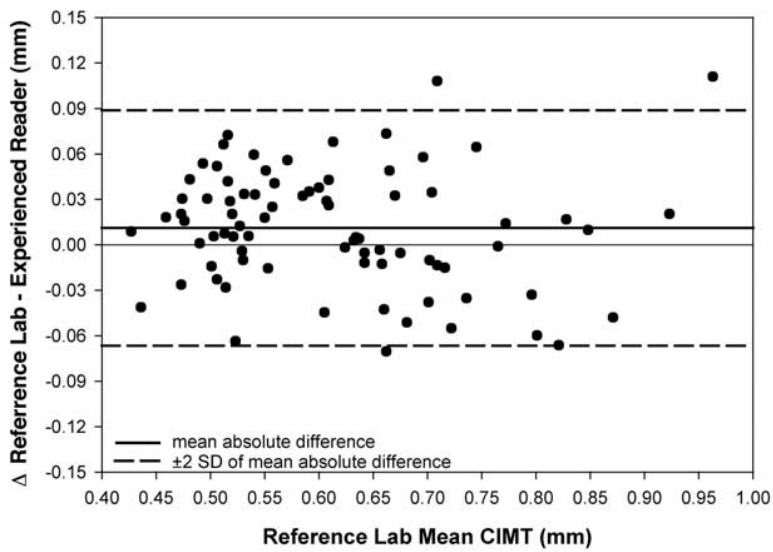

Figure 2 Bland-Altman plot comparing mean common carotid artery intima-media thickness (CIMT) values with the reference laboratory for experienced reader.

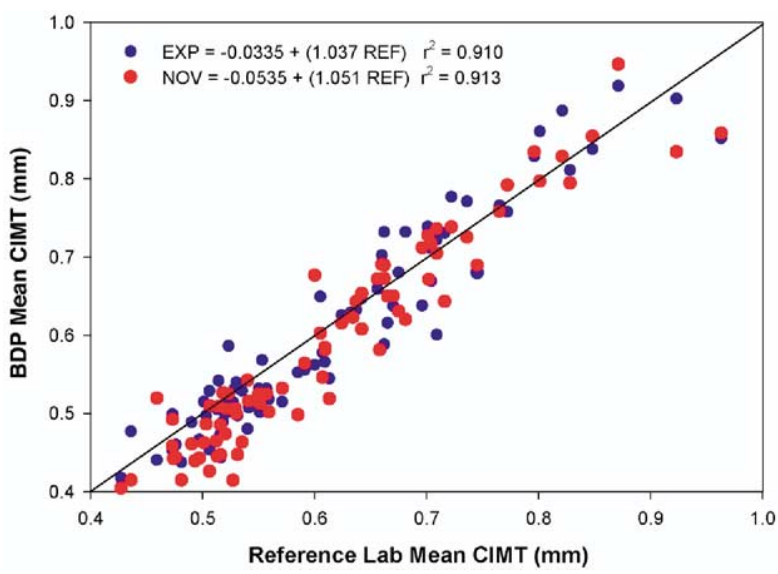

Figure 3 Comparison of mean common carotid artery intima-media thickness (CIMT) values using border detection program $(B D P)$ and reference laboratory $(R E F) . E X P$, Experienced reader; $N O V$, novice reader.

lent $\left(P_{\mathrm{TOST}}<.05\right)$ to the REF laboratory (Table 1$)$. The mean CIMT measurements by the EXP reader correlated strongly with the measurements by the REF laboratory $(r=0.954, P<.001)$ with a mean absolute difference of $0.011 \pm 0.004 \mathrm{~mm}$ (Figures 2 and 3). Maximum CIMT values correlated strongly $(r=0.914, P<.001)$ with a mean absolute difference of $-0.031 \pm 0.007 \mathrm{~mm}$ (Figure 4). Similarly, the NOV reader's mean absolute differences compared with the REF laboratory were small, and measurements were strongly correlated for mean CIMT (mean absolute difference $=0.022 \pm 0.004 \mathrm{~mm}, r=$ $0.955, P_{\text {correlation }}<.001$ ) (Figures 3 and 5) and maximum CIMT $(-0.011 \pm 0.006 \mathrm{~mm}, r=0.921$, $\left.P_{\text {correlation }}<.001\right)$ (Figure 4). 


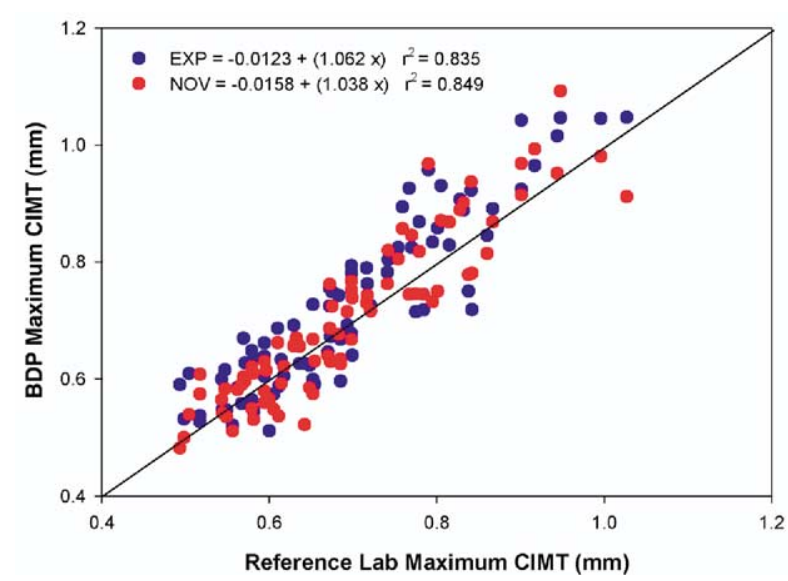

Figure 4 Comparison of maximum common carotid artery intima-media thickness (CIMT) values using border detection program $(B D P)$ and reference laboratory. EXP, Experienced reader; $N O V$, novice reader.

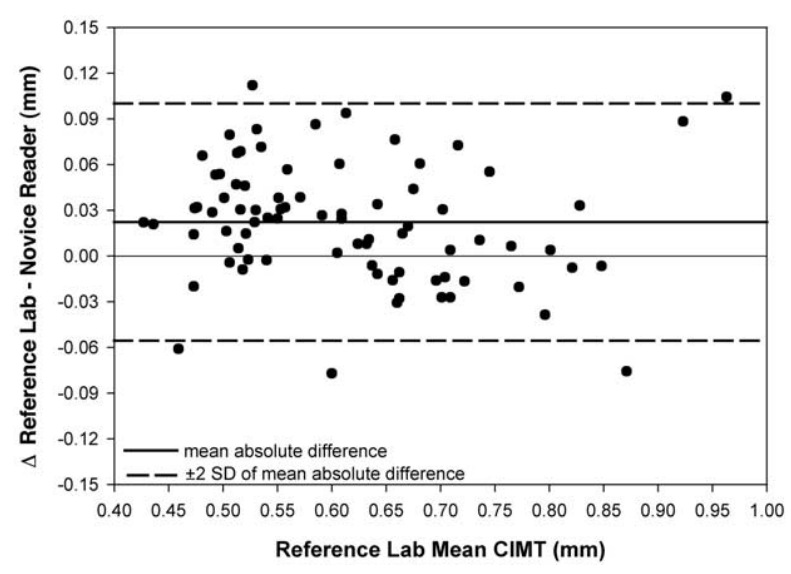

Figure 5 Bland-Altman plot comparing mean common carotid artery intima-media thickness (CIMT) values with reference laboratory novice reader.

\section{Intraobserver Reproducibility}

Intraobserver reproducibility of mean and maximum CIMT measurements using BDP was high for both EXP and NOV readers (Table 2). As expected, it was stronger for the EXP reader. Repeated mean CIMT measurements by the EXP reader correlated strongly $(r=0.979, P<.001)$ with a nonsignificant mean absolute difference of $0.003 \pm 0.003 \mathrm{~mm}$ and a CV of $3.1 \%$. Maximum CIMT readings by the EXP reader also correlated strongly $(r=0.968, P<.001)$ with a mean absolute difference of $0.009 \pm 0.004 \mathrm{~mm}$ and a $\mathrm{CV}$ of $3.6 \%$. For the NOV reader using BDP, repeated mean CIMT measurements also correlated strongly and had convincing correlations $(r=0.927, P<.001)$ with a mean absolute difference of $-0.040 \pm 0.005 \mathrm{~mm}(\mathrm{CV}=7.8 \%)$. Maximum CIMT readings by the NOV reader also
Table 2 Intraobserver reproducibility for mean and maximum common CIMT thickness measurements

\begin{tabular}{ccc}
\hline & Experienced reader & Novice reader \\
\hline $\begin{array}{c}\text { Mean CIMT } \\
\text { Mean absolute } \\
\text { difference, } \mathrm{mm}\end{array}$ & $0.003 \pm 0.003$ & $-0.040 \pm 0.005$ \\
$\begin{array}{c}\left.\text { ( } p_{\text {correlation }}\right) \\
\text { Coefficient of } \\
\text { variation }\end{array}$ & $.979(<.001)$ & $.927(<.001)$ \\
$\begin{array}{c}\text { Maximum CIMT } \\
\text { Mean absolute } \\
\text { difference, } m \text { mm }\end{array}$ & $0.009 \pm 0.004$ & $-0.021 \pm 0.006$ \\
$\begin{array}{c}\left.\text { (P } p_{\text {correlation }}\right) \\
\text { Coefficient of } \\
\text { variation }\end{array}$ & $0.968(<0.001)$ & $0.938(<0.001)$ \\
\hline
\end{tabular}

CIMT, Carotid intima-media thickness.

correlated strongly $(r=0.938, P<.001)$ with a mean absolute difference of $-0.021 \pm 0.006 \mathrm{~mm}$ $(\mathrm{CV}=5.7 \%)$.

\section{Measurement Times}

The reading speed of the NOV reader increased after the first set of measurements. The average per-participant reading time during the first set of measurements was $20.8 \pm 0.6$ minutes. It decreased to $18.6 \pm 0.6$ $\mathrm{min} /$ participant $(P=.031)$ during the second set of measurements. The EXP reader did not have a significant difference between measurement times but the average time was significantly lower than for the NOV reader $(6.9 \pm 0.3 \mathrm{~min} /$ participant, $P<$ .001).

\section{DISCUSSION}

Ultrasound measurement of CIMT is a safe and effective technique for assessing atherosclerosis burden and determining cardiovascular risk. ${ }^{7}$ Although CIMT has been recommended by the American Heart Association to detect subclinical atherosclerosis, its use in an office setting has been limited by the need to accurately identify and measure small but clinically meaningful differences in carotid wall thickness. In addition, a highly standardized protocol is needed to ensure that measurements are reproducible and representative of the degree of atherosclerosis. These requirements can make the assessment of CIMT time-consuming, a problem that is compounded by potentially expensive instrumentation. $^{7}$ To translate the compelling research data supporting CIMT as a risk prediction tool into a clinical setting, software that enables users to make accurate and reproducible CIMT measurements rapidly is necessary. This software could be incorporated into the ultrasound system or readily transmitted to a personal computer. 
In this study, both EXP and NOV readers obtained very reproducible measurements of mean and maximum CIMT values using the new BDP. As expected, the reproducibility of the EXP reader was better than that of the NOV reader; however, CV for the measurements using BDP were excellent and comparable with other studies. ${ }^{9,14,15}$ Measurements by both NOV and EXP readers correlated strongly with those of the REF laboratory with minimal systematic bias, and bioequivalence was demonstrated. BlandAltman plots and linear regression models for mean and maximum CIMT values successfully illustrate the striking similarity between the readers using BDP and the REF laboratory, and high intrareader repeatability. The improvement in the NOV reader's efficiency with repeated use of BDP also demonstrates the ease with which this program can be mastered and used effectively. A similar study that compared measurement times using a different semiautomated BDP to manual tracing methods found significant reductions in reading times of nearly 50\% for a NOV reader. ${ }^{15}$

The EXP reader edited approximately $20 \%$ of the tracings by BDP. The NOV reader modified approximately $33 \%$ the BDP tracings. Approximately half were edited to make sure that the edges of the measurement borders corresponded precisely to the segment definition used in this study. The rest were to modify the blood-intima and media-adventitia interfaces. Modification of the detected border was more common when carotid artery image deviated from horizontal and for images that were not at the optimal angle of interrogation.

Strengths of this study include blinded, direct comparisons between measurements using the BDP and a well-established core REF laboratory demonstrating measurement accuracy, and reproducibility by both a NOV and EXP reader similar to or better than that reported in the literature. . $^{94,15}$ This study only evaluated the far wall of the CCA, a relatively straight segment that is easier to image than other segments of the carotid artery. These characteristics favor better performance of BDP, but also are more practical if CIMT is to be done in an office setting, where longer, more comprehensive protocols used in clinical trials may not be feasible. Of note, far-wall CIMT and its changes have been associated with cardiovascular risk and used to measure response to therapy in clinical trials. ${ }^{3,16}$ Because there were fewer participants at the high end of the CIMT range, greater differences between the techniques for high or extreme CIMT values cannot be excluded. Incorporation of a BDP into an inexpensive lightweight or portable ultrasound system, validation of an abbreviated scanning protocol for officebased cardiovascular risk assessment, and studies demonstrating improved cardiovascular outcomes using CIMT measurements are needed to continue to facilitate clinical use of CIMT measurements.

\section{Conclusions}

CIMT measurements using this new, semiautomated BDP were accurate and reproducible. The userfriendly software interface enabled a NOV reader with no ultrasound training to master the program quickly and effectively. The combination of easy-touse software and the ability to reproduce small measurements make this BDP appear suitable for use in an office setting.

The authors express their appreciation to Roger Brown, $\mathrm{PhD}$, for statistical assistance and to Ward A. Riley, PhD, for assistance with study design and reference measurements.

\section{REFERENCES}

1. Mitchell JR, Schwartz CJ. Relationship between arterial disease in different sites: a study of the aorta and coronary, carotid, and iliac arteries. Br Med J 1962;5288:1293-301.

2. Young W, Gofman J, Tandy R, Malamud N, Waters E. The quantitation of atherosclerosis III: the extent of correlation of degrees of atherosclerosis with and between the coronary and cerebral vascular beds. Am J Cardiol 1960;8:300-8.

3. Hodis H, Mack W, LaBree L, Selzer R, Liu C, Liu C, et al. The role of carotid arterial intima-medial thickness in predicting clinical coronary events. Ann Intern Med 1998;128:262-9.

4. Burke G, Evans G, Riley W, Sharrett A, Howard G, Barnes R, et al. Arterial wall thickness is associated with prevalent cardiovascular disease in middle-aged adults: the atherosclerosis risk in communities (ARIC) study. Stroke 1995;26:386-91.

5. Chambless LE, Heiss G, Folsom AR, Rosamond W, Szklo M, Sharrett AR, et al. Association of coronary heart disease incidence with carotid arterial wall thickness and major risk factors: the atherosclerosis risk in communities (ARIC) study, 1987-1993. Am J Epidemiol 1997;146:483-94.

6. Chambless LE, Folsom AR, Clegg LX, Sharrett AR, Shahar E, Nieto FJ, et al. Carotid wall thickness is predictive of incident clinical stroke: the atherosclerosis risk in communities (ARIC) study. Am J Epidemiol 2000;151:478-87.

7. Greenland P, Abrams J, Aurigemma GP, Bond MG, Clark LT, Criqui $\mathrm{MH}$, et al. Prevention conference V: beyond secondary prevention; identifying the high-risk patient for primary prevention-noninvasive tests of atherosclerotic burden, writing group III. Circulation 2000;101:E16-22.

8. Bond $\mathrm{M}$, Barnes R, Riley $\mathrm{W}$, Wilmoth S, Chambless L, Howard G, et al. High-resolution B-mode ultrasound scanning methods in the atherosclerosis risk in communities study (ARIC). J Neuroimaging 1991;1:68-73.

9. Riley WA, Barnes RW, Applegate WB, Dempsey R, Hartwell $\mathrm{T}$, Davis VG, et al. Reproducibility of noninvasive ultrasonic measurement of carotid atherosclerosis: the asymptomatic carotid artery plaque study. Stroke 1992;23:1062-8.

10. Bland JM, Altman DG. Measurement error proportional to the mean. BMJ 1996;313:106.

11. Bland JM, Altman DG. Statistical methods for assessing agreement between two methods of clinical measurement. Lancet 1986;1:307-10. 
12. Hsu J, Hwang J, Liu H-K, Ruberg S. Confidence intervals associated with tests for bioequivalence. Biometrika 1994;81: 103-14.

13. Chow S, Liu J. Design and analysis of bioequivalence studies. New York: Marcel Dekker; 1992.

14. Kanters SD, Algra A, van Leeuwen MS, Banga JD. Reproducibility of in vivo carotid intima-media thickness measurements: a review. Stroke 1997;28:665-71.

15. Stein JH, Korcarz CE, Mays ME, Douglas PS, Palta M, Zhang $\mathrm{H}$, et al. A semi-automated border detection program that facilitates clinical use of ultrasound carotid intima-media thickness measurements. J Am Soc Echocardiogr 2005; 18:244-51.

16. Taylor AJ, Sullenberger LE, Lee HJ, Lee JK, Grace KA. Arterial biology for the investigation of the treatment effects of reducing cholesterol (ARBITER) 2: a doubleblind, placebo-controlled study of extended-release niacin on atherosclerosis progression in secondary prevention patients treated with statins. Circulation 2004;110: 3512-7. 